\title{
Strong reduction of the coercivity by a surface acoustic wave in an out-of-plane magnetized epilayer
}

\author{
L. Thevenard, ${ }^{1,{ }^{*}}$ I. S. Camara, ${ }^{1}$ J.-Y. Prieur, ${ }^{1}$ P. Rovillain, ${ }^{1}$ A. Lemaître, ${ }^{2}$ C. Gourdon,,${ }^{1}$ and J.-Y. Duquesne ${ }^{1}$ \\ ${ }^{1}$ Sorbonne Universités, UPMC Université Paris 06, CNRS, Institut des Nanosciences de Paris, 4 place Jussieu, 75252 Paris, France \\ ${ }^{2}$ Laboratoire de Photonique et Nanostructures, CNRS, UPR 20, Route de Nozay, 91460 Marcoussis, France
}

(Received 5 January 2016; revised manuscript received 1 March 2016; published 11 April 2016)

\begin{abstract}
Inverse magnetostriction is the effect by which magnetization can be changed upon application of stress/strain. A strain modulation may be created electrically by exciting interdigitated transducers to generate surface acoustic waves (SAWs). Hence SAWs appear as a possible route towards induction-free undulatory magnetic data manipulation. Here we demonstrate experimentally on an out-of-plane magnetostrictive layer a reduction of the coercive field of up to $60 \%$ by a SAW, over millimetric distances. A simple model shows that this spectacular effect can be partly explained by the periodic lowering of the strain-dependent domain nucleation energy by the SAW. This proof of concept was done on $(\mathrm{Ga}, \mathrm{Mn})(\mathrm{As}, \mathrm{P})$, a magnetic semiconductor in which the out-of-plane magnetic anisotropy can be made very weak by epitaxial growth; it should guide material engineering for all-acoustic magnetization switching.
\end{abstract}

DOI: 10.1103/PhysRevB.93.140405

The use of electrical voltage-rather than current-is a promising approach to limit heat dissipation and facilitate device size reduction for magnetic data manipulation. Static changes to the anisotropy by carrier density modification, magnetoelectricity, or magnetoelasticity (mediated by piezoelectricity) have been demonstrated via gating in metals [1-3] and magnetic semiconductors [4-7]. For instance, piezoelectric actuators have enabled the fieldless driving of domain walls in the highly magnetostrictive $\mathrm{FeGa}$ [3], and the static manipulation of magnetization in (Ga,Mn)As [7].

Using magnetoelasticity with a time-varying strain, such as surface acoustic waves (SAWs), or bulk picosecond acoustic pulses (PAPs), could allow faster switching. Their wave properties would also offer the possibility of focusing or interference to switch magnetization selectively, a potentially exciting perspective for magnetic data storage applications. SAWs and PAPs have been shown to couple to magnetization in magnetostrictive materials [8-11]. Relying on this coupling, recent work has predicted $[12,13]$ and demonstrated experimentally [14] that acoustic waves can trigger resonant precessional switching. SAWs can moreover assist-nonresonantly - the coherent reversal of small structures [15,16], or in larger samples could trigger the nucleation or propagation of domain walls $[13,17]$. In that case, the lever is expected to be the dependency of the domain wall energy on the magnetoelastic constant. So far the strongest reported effect has been in thin FeGa layers, in which a $11 \%$ reduction of the coercive field by SAW was observed, as well as early evidence of localized magnetization reversal assisted by a focused SAW [18,19]. No particular mechanism was identified, however. In this Rapid Communication, we extend this proof of concept to out-of-plane magnetized materials, as well as to an entirely different class of materials: dilute magnetic semiconductors (DMSs). We evidence an increased efficiency (over 50\% reduction of the coercive field), and show by a simple model that SAW-assisted nucleation is partly responsible for this effect.

\footnotetext{
*thevenard@insp.jussieu.fr
}

Archetype among DMSs, (Ga,Mn)As and its recently developed alloy $(\mathrm{Ga}, \mathrm{Mn})(\mathrm{As}, \mathrm{P})$ have a carrier-mediated ferromagnetic phase which renders their magnetic anisotropy sensitive to both static and dynamic strain $[7,20,21]$. Rayleigh waves are particularly well suited to the magnetoelasticity of $(\mathrm{Ga}, \mathrm{Mn})(\mathrm{As}, \mathrm{P})$, since their strain components directly couple to the uniaxial out-of-plane anisotropy. SAW-induced precessional switching was, for instance, recently demonstrated [14], under fields of a few tens of $\mathrm{mT}$ applied perpendicular to the easy axis. In this work, we focus on the geometry where the field is along the easy axis, and use Kerr microscopy to monitor modifications to the coercive field and the shape of nucleated domains in the presence of a SAW.

The sample studied is an out-of-plane magnetized $d=50$ $\mathrm{nm}$ thick layer of $\left(\mathrm{Ga}_{1-x}, \mathrm{Mn}_{x}\right)\left(\mathrm{As}_{0.96}, \mathrm{P}_{0.04}\right)$ in tensile strain on GaAs with an active Mn concentration $x_{\mathrm{Mn}}^{\mathrm{eff}} \approx 3.5 \%$, and a Curie temperature of $T_{C}=95 \mathrm{~K}$. The SAWs were generated and detected by two opposite sets of gold interdigitated transducers (IDTs) evaporated on a $\mathrm{SiO}_{2} / \mathrm{ZnO}$ bilayer, with a $1 \mathrm{~mm}$ aperture. The IDT teeth were $1.25 \mu \mathrm{m}$ wide and equidistant, giving a SAW wavelength of $5 \mu \mathrm{m}$ and frequency of $f_{\mathrm{SAW}}=549 \mathrm{MHz}$ (SAW period $\left.T_{\mathrm{SAW}} \approx 1.8 \mathrm{~ns}\right)$. A window etched in the $\mathrm{ZnO}$ layer between the two IDTs facilitated the Kerr imaging, with the $\mathrm{SiO}_{2}$ layer thickness adjusted for an optimal Kerr contrast [22] at $\lambda=600 \mathrm{~nm}$. Whereas the IDTs are separated by $2 \mathrm{~mm}$, the typical field of view for the polar Kerr microscope is between 360 and $994 \mu \mathrm{m}$ wide, depending on the magnification used [Fig. 1(a)]. To limit device breakdown at high powers, the SAW is generated in bursts (typically $\tau_{\mathrm{SAW}}=600 \mathrm{~ns}$ long) emitted at a low repetition frequency (typically $f_{\text {rep }}=50 \mathrm{~Hz}$ ). The maximum surface strain has been estimated using a vector network analyzer (VNA) to be of $6.2 \times 10^{-4}$. The magnetic field is applied in plateaus of a few seconds to allow image acquisition and transfer [see Fig. 1(b) for the time dependence of the strain]. Spatially averaging the Kerr intensity at each field gives the hysteresis cycle, from which we define the coercive field $B_{c}$ as the one giving zero average magnetization.

Without SAW, the hysteresis loop averaged in front of the emitting IDT is square [Fig. 1(c)], and the reversal is driven by multiple nucleations across the layer. Note that this 

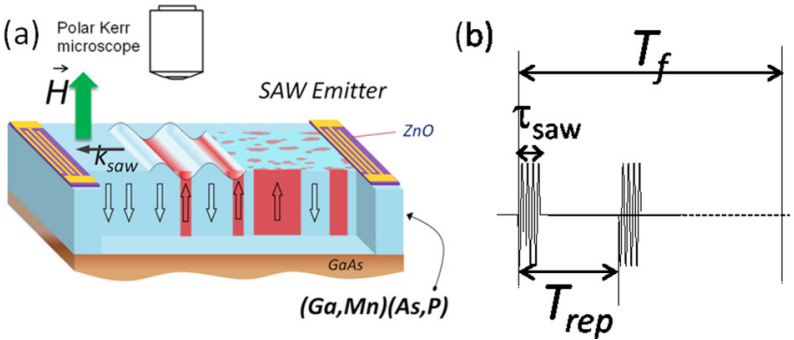

\section{(c) Without SAW}

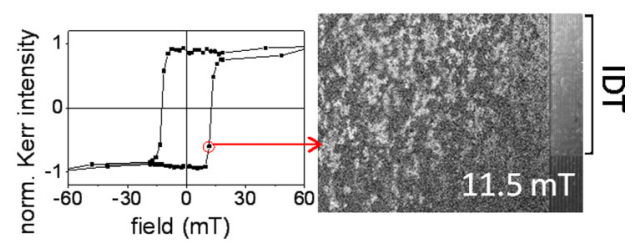

FIG. 1. (a) Schematics of the experimental setup (not to scale). A thin $\mathrm{SiO}_{2}$ layer (not represented) is deposited on the (Ga,Mn)(As,P). The SAW assists domain nucleation in the half period during which it lowers the DW energy. (b) Time dependence of the strain (not to scale). SAWs are generated in bursts of duration $\tau_{\mathrm{SAW}}=600 \mathrm{~ns}$, every $T_{\text {rep }}=20 \mathrm{~ms}$, during the entire field plateaus of length $T_{f}=4 \mathrm{~s}$. (c) Hysteresis cycle averaged in front of the emitting IDT without SAW $(T=10 \mathrm{~K})$ and Kerr microscopy image $\left(365 \times 273 \mu \mathrm{m}^{2}\right)$ of the domains during the field reversal. The magnetic part of the image has been divided by a reference image taken at remanence and the rightmost part left raw to show the transducer.

behavior is very different from high anisotropy (Ga,Mn)As samples [23], in which nucleation events are rare, especially at low temperatures, and occur at high enough fields to then trigger the fast reversal of the whole layer. A spatial mapping of the coercive field does not highlight any particular nucleation spots. Hysteresis cycles are then taken with the SAW "on." Figure 2(a) shows, for instance, data taken at $30 \mathrm{~K}$ when exciting the IDT at its resonance frequency $549 \mathrm{MHz}$ with an incident $\mathrm{rf}$ power $P_{0}=8.9 \mathrm{~W} / \mathrm{mm}$, and a repetition rate of $f_{\text {rep }}=50 \mathrm{~Hz}$. The coercive field in front of the emitting IDT is now $4.3 \mathrm{mT}$, reduced by over $50 \%$ compared to the one without SAW (9.8 mT). The Kerr images show that switching clearly occurs first on the SAW path [Figs. 2(b)-2(e)]. More specifically, it initiates at low field $(<3.5 \mathrm{mT})$, on the edge of the wave front [Fig. 2(b)] and with sparse nucleation centers above. At $8 \mathrm{mT}$, the layer has almost fully switched on the SAW path, but not at all out of it. As the field is further increased to $9.5 \mathrm{mT}$ [Fig. 2(f)], switching is finally triggered out of the SAW path and the magnetization fully reverses at $11 \mathrm{mT}$.

In order to have a first hint as to the reversal mechanism, a magnetic aftereffect experiment is performed, i.e., a study of the time dependence of the magnetic susceptibility after a given initialization. After saturating the layer "up," a field of opposite sign is applied, low enough to allow slow magnetization reversal $(B=2 \mathrm{mT}, T=30 \mathrm{~K})$. Three experiments are then run under different SAW repetition frequencies $\left(f_{\text {rep }}=15\right.$, 20 , and $30 \mathrm{~Hz}$ ). For each $f_{\text {rep }}$, the rf power is applied long enough to total the passage of 6000 SAW bursts. The intensity is averaged in front of the IDT, normalized to its initial value, $I(t) / I(t=0)$, and plotted versus the number of SAW bursts [inset of Fig. 2(a)]. The three curves have a very similar shape and final fractional reversed magnetization (75\%) regardless of the repetition frequency. This result suggests a cumulative effect necessary for the switching, given by the total number of SAW periods with a weak influence of the repetition rate.

The excitation power was then varied over two decades [Fig. 3(a), $T=10 \mathrm{~K}$ ]. The SAW efficiency clearly decreases with decreasing power, with the coercive field recovering its SAW-less value at $0.01 P_{0}$ (cycle not shown). The final $B_{c}(P)$ curve is roughly linear in power [Fig. 6(a)], and exciting SAWs with the left IDT gives an identical trend. No $B_{c}$ reduction is observed when exciting the IDT off resonance, for which no SAW is generated [rf power at $570 \mathrm{MHz}$, open symbols in Fig. 3(a)], proving the effect is not due to the field radiated by the antenna. To obtain the spatial dependence of the coercive field, the Kerr intensity is averaged over $10 \times 10$ pix $^{2}$ $\left(6.6 \times 6.6 \mu \mathrm{m}^{2}\right)$ bins for each field. The resulting bin-specific hysteresis cycles are then analyzed numerically to extract a local coercive field which is overlaid on the original image as a color map, where blue (red) indicates a poor (good)

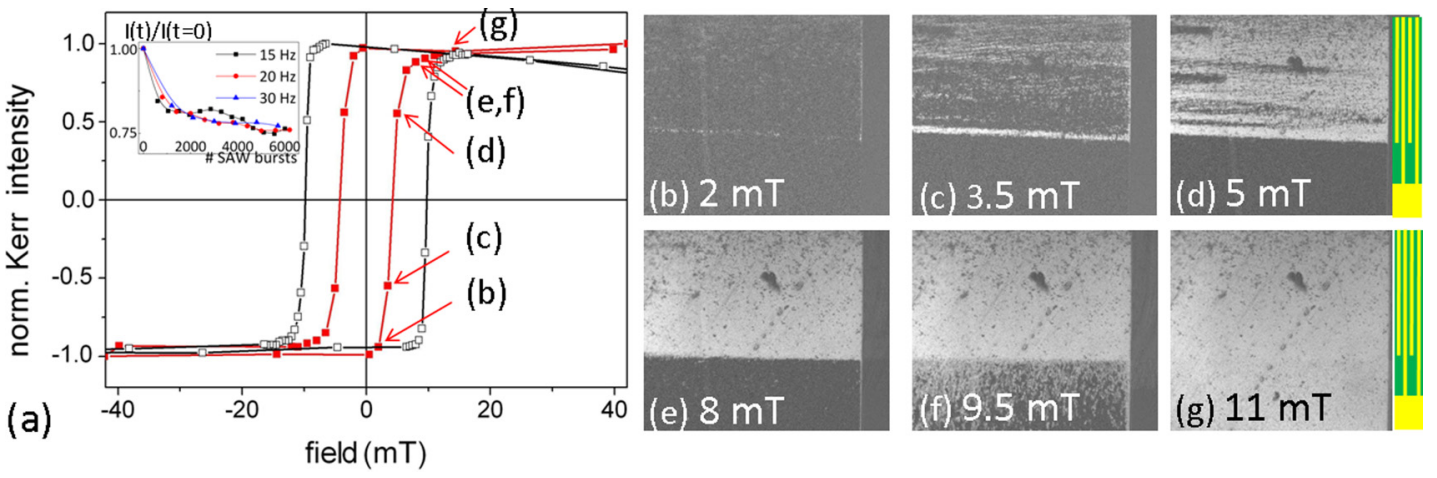

FIG. 2. SAW-assisted reversal at $P_{0}=8.9 \mathrm{~W} / \mathrm{mm}(T=30 \mathrm{~K})$ : (a) Hysteresis cycles averaged in front of the SAW emitter, with and without SAW. Inset: Magnetic aftereffect experiment at $B=2 \mathrm{mT}$ under $6000 \mathrm{SAW}$ bursts, corresponding to a duration of $6 \mathrm{~min}\left(f_{\text {rep }}=15 \mathrm{~Hz}\right)$, $5 \mathrm{~min}\left(f_{\text {rep }}=20 \mathrm{~Hz}\right)$, or $3.5 \mathrm{~min}\left(f_{\text {rep }}=30 \mathrm{~Hz}\right)$, intensity averaged in front of the IDT normalized to its initial value. (b)- $(\mathrm{g})$ Kerr microscopy images $\left(690 \times 924 \mu \mathrm{m}^{2}\right)$ corresponding to the cycle under SAW in (a). 


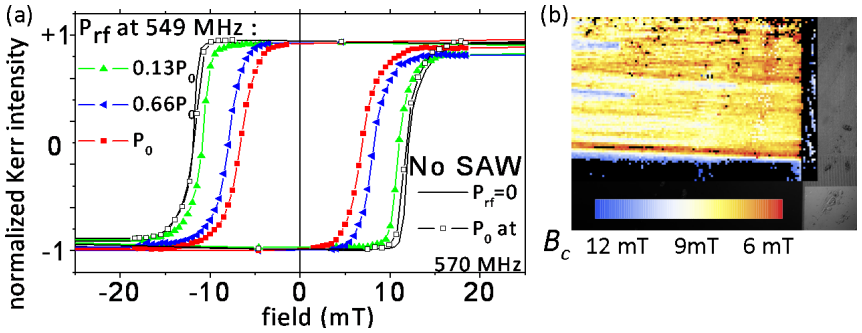

FIG. 3. (a) Hysteresis cycles averaged on the SAW path $(T=$ $10 \mathrm{~K}$ ), without SAW (solid line), off IDT resonance at $P_{0}$ (open symbols), and at the IDT resonance for different powers. (b) Spatial map of the coercive field based on the hysteresis cycle taken at $P_{0}$ (image size $690 \times 924 \mu \mathrm{m}^{2}$ ). Blue (red) indicates a poor (good) SAW efficiency. Transparent bins indicate that the data were locally too poor to extract a coercive field.

SAW efficiency [Fig. 3(b)]. The SAW efficiency is clearly not uniform, because of both a complex wave-front shape due to the finite aperture of the IDT, and defects on the sample. Large defects on the surface of the sample "shadow" the SAW (upper left-hand corner), giving a higher (bluer) coercive field in their wake. The resulting spatial dispersion of the SAW efficiency also explains the broadening of the switching step with rf power seen in the hysteresis cycles averaged over the whole SAW path [Fig. 3(a)].

The efficiency of this coercivity reduction was then studied as a function of temperature. Hysteresis cycles were taken up to $80 \mathrm{~K}\left(15 \mathrm{~K}\right.$ below $\left.T_{C}\right)$, beyond which the Kerr contrast was too poor for proper analysis (Fig. 4). The efficiency of the SAW-assisted reversal is roughly constant up to $38 \mathrm{~K}(5-6 \mathrm{mT}$ reduction of $B_{c}$ ) and then decreases, before canceling out at about $70 \mathrm{~K}$. This may be due to a concomitant decrease of the IDT's electromechanical conversion efficiency. This was indeed observed using a VNA. Finally, we studied the distance up to which the SAW assists magnetization reversal $(T=$ $10 \mathrm{~K})$. Three hysteresis cycles were taken, moving for each one the sample in front of the objective so as to observe the whole $2 \mathrm{~mm}$ long delay line between emitting and receiving transducers while the SAW was "on." The resulting stitched panorama shows that the SAW stays efficient over millimetric distances (Fig. 5).

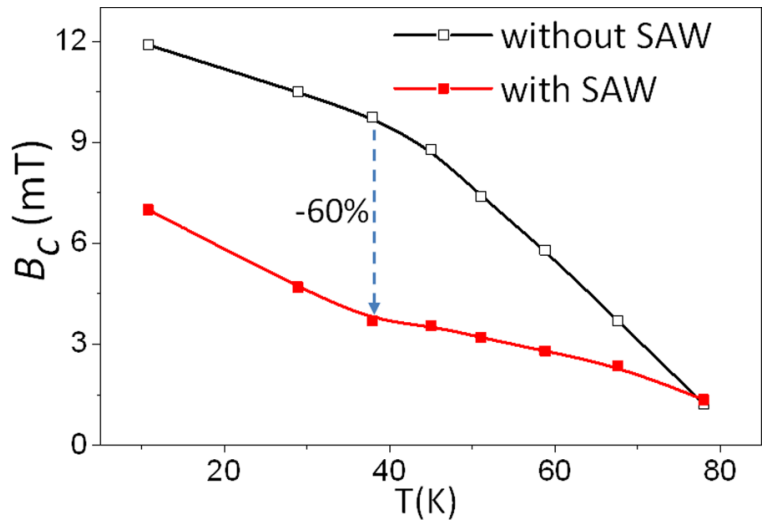

FIG. 4. Temperature dependence of the coercive field averaged in front of the IDT (incident rf power $P_{0}$ ).

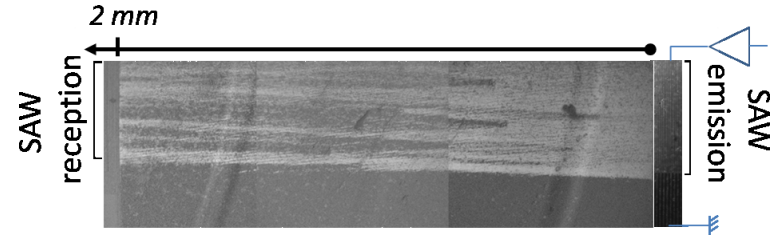

FIG. 5. Kerr microscopy images $\left(690 \times 924 \mu \mathrm{m}^{2}\right)$ taken at $P_{0}$, $T=10 \mathrm{~K}$, and $B=11 \mathrm{mT}$. The vertical streaks in the first and last images are microscopy artifacts.

We now review the possible mechanisms responsible for the spectacular effect of the surface acoustic wave on the coercivity. We first discuss why thermal effects can be discarded. Indeed, rf excitation of an IDT can possibly generate heat because of the Joule effect in the transducer or Foucault electrical losses in the metallic bodies submitted to the radiated rf electromagnetic fields. The spectrum of thermal excitation will comprise a dc component (due to the mean input power) and high frequency components in the $\mathrm{GHz}$ range (twice the $\mathrm{rf}$ frequency). The dc component will be isotropic and therefore cannot account for the observed highly directive magnetization reversal. Moreover, the dc component depends on the repetition rate so that it can be monitored: Measuring the acoustic velocity change versus repetition rate, the temperature rise was estimated to be under $0.5 \mathrm{~K}$ (for a base temperature of $30 \mathrm{~K}$ ). High frequency components will exhibit very short diffusion lengths, in the $\mu \mathrm{m}$ range depending on diffusivities, and can therefore not account for the observed effect over millimetric distances. The acoustic wave could also induce a temperature rise while propagating because part of the acoustic loss results in incoherent phonons production (thermoelastic, Akhieser effects [24], etc.). This effect is in fact usually negligible. In our case, crudely assuming that the entire lost acoustic energy is dissipated as heat, we estimate that the temperature rise due to those processes lies below $0.05 \mathrm{~K}$.

We therefore focus on nonthermal effects of the SAW. With the field along the easy axis, magnetization reversal proceeds by the nucleation and propagation of domain walls (DWs). With $A_{\text {ex }}$ the exchange constant [25] and $K_{u}$ the out-ofplane uniaxial anisotropy [measured by cavity ferromagnetic resonance (FMR)], their energy is given by $\sigma=4 \sqrt{A_{\mathrm{ex}} K_{u}}$, and their velocity in the stationary regime [26] by $v_{\text {stat }} \propto \Delta$, with $\Delta=\sqrt{A_{\mathrm{ex}} / K_{u}}$. Both $\sigma$ and $v_{\text {stat }}$ depend on $K_{u}$, the dominant magnetoelastic term in $(\mathrm{Ga}, \mathrm{Mn})(\mathrm{As}, \mathrm{P})$. The SAW could thus-in theory-affect both steps of the reversal. It has been argued, however [16], that the high frequencies of SAWs $(>100 \mathrm{MHz})$ would not allow enough time for the relatively slow DW propagation (speeds of tens of $\mathrm{ms}^{-1}$ in GaMnAs [27])—unless particular geometries are used [17] so we focus on their effect on nucleation. The concavity of the magnetic aftereffect curves taken under SAW [inset of Fig. 2(a)] supports a nucleation-dominated reversal [28]. We therefore model the influence of the SAW on domain nucleation, and how it may lead to the power dependence of the coercivity reduction observed in Fig. 6(a).

Following Hubert et al. [29], the nucleation of an isolated domain of radius $r_{\text {nuc }}$ is driven by the competition between the cost to create a DW, $\Delta E_{\text {nuc }}=2 \pi r_{\text {nuc }} d \sigma$, and the associated 


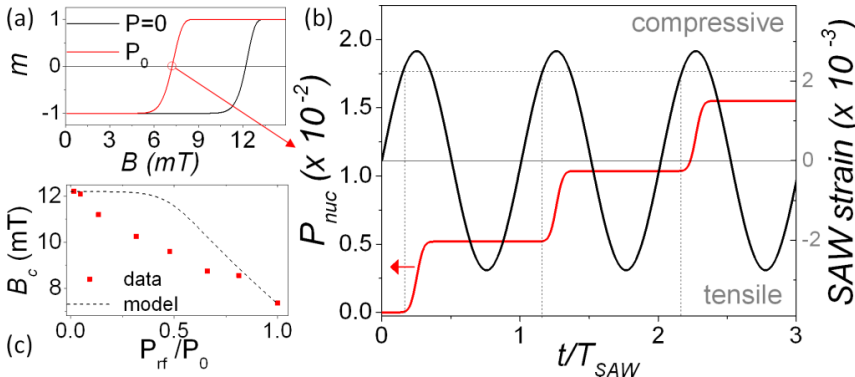

FIG. 6. (a) Fraction of reversed magnetization vs field calculated by the model, with and without SAW. (b) Time dependence of the SAW-induced strain and nucleation probability at $B=7.35 \mathrm{mT}$. Dotted lines materialize the onset of nucleation triggered by reaching a threshold compressive strain. (c) Power dependence of the coercive field at $T=10 \mathrm{~K}$, nucleation model, and data, taken as the central value of the Gaussian distribution of $B_{c}$ in a small area in front of the IDT.

lowering of the Zeeman energy [30] $\Delta E_{Z}=-2 M_{s} B d \pi r_{\text {nuc }}^{2}$, with $\Delta E_{\text {tot }}=\Delta E_{Z}+\Delta E_{\text {nuc. }}$. We remind that $d$ is the layer thickness. This nucleation is thermally activated, with typical switching times given by $\tau=\tau_{0} \exp \left[\frac{\Delta E_{\text {tot }}}{k_{B} T}\right], 1 / \tau_{0}$ being an attempt frequency [31] ( $\left.\tau_{0} \approx 10 \mathrm{ps}\right)$. The so-called "droplet model" [32] argues that the nucleation radius is well approximated by the one leading to $\frac{\partial \Delta E_{\text {tot }}}{\partial r_{\text {nuc }}}=0$, i.e., $r_{\text {nuc }}=$ $\frac{\sigma}{2 B M_{s}}$. This allows us to rewrite the total energy barrier as $\Delta E_{\mathrm{tot}}=\frac{\pi d \sigma^{2}}{2 B M_{s}}$. In our particular case, the DW energy is time dependent, with $\sigma(t)=4 \sqrt{A_{\mathrm{ex}} K_{u}(t)} . K_{u}(t)=-\frac{M_{s} A_{2 \varepsilon}}{2}\left[\varepsilon_{0}+\right.$ $\varepsilon(t)]$ is a function of the static and dynamic (SAW-induced) strains, with $\varepsilon_{0}=\varepsilon_{z z, 0}-\varepsilon_{x x, 0}=-0.161 \%, A_{2 \varepsilon}=34 \mathrm{~T}$ [9], and $\varepsilon(t)=\varepsilon_{Z Z}(t)-\frac{\varepsilon_{X X}(t)}{2}=\varepsilon_{\mathrm{SAW}} \sin \omega_{\mathrm{SAW}} t$. The layer being much thinner than the SAW wavelength, we have assumed the strain constant across it. Moreover, we have dropped the in-plane spatial dependence of $\varepsilon(t)$ since the SAW is propagating and not stationary. The DW nucleation energy barrier is then time dependent so that the probability to nucleate a domain during a field plateau of duration $T_{f}$ is given by

$$
P_{\text {nuc }}\left(T_{f}, B\right)=1-\exp \left(-\int_{0}^{T_{f}} \exp \left[-\frac{\Delta E_{\mathrm{tot}}(t, B)}{k_{B} T}\right] \frac{d t}{\tau_{0}}\right) .
$$

If the reversal is indeed mainly driven by nucleation, and that these nucleation sites do not interact, the probability $P_{\text {nuc }}=0.5$ to nucleate at a given site is then equivalent to reversing half the total magnetization: $m=M(B) / M_{s}=0$ with $m(B)=1-2 P_{\text {nuc }}(B)$. Using the sample's $10 \mathrm{~K}$ micromagnetic parameters, we calculate numerically $m(B)$ for each field of the cycle [Fig. 6(b)], and define the coercive field by $m\left(B_{c}\right)=0$. Since the energy barrier is several $\mathrm{eV}$ high, much larger than the thermal energy, we assume as others [33] a localized lowering of the DW energy $\sigma^{*}=\gamma \sigma$ due to defects or magnetic anisotropy inhomogeneities. In the absence of SAW $\left[P_{\mathrm{rf}}=0\right.$, Figs. 6(a) and 6(b)], $B_{c, 0}=12 \mathrm{mT}$ yields $\gamma=5.4 \times 10^{-3}$. This corresponds to a nucleation radius of $r_{\text {nuc }}=\frac{\sigma^{*}}{2 B M_{s}}=5.5 \mathrm{~nm}$, i.e., of the order of the DW width $\Delta \approx 6 \mathrm{~nm}$ at $10 \mathrm{~K}$. This value is kept for the calculation in the presence of SAW.
We then implement numerically the experimental SAW time dependence [Fig. 1(b)], and take the SAW amplitude as [34] $\varepsilon_{\mathrm{SAW}}=\eta \sqrt{P_{\mathrm{rf}}} . \eta$ is a measure of the electromechanical conversion efficiency, and of the impedance matching of our system. It is linear with injected rf power up to $P_{0}$ and is then the only unknown parameter; its value is adjusted so as to give the observed coercive field at a given rf power. For instance, running the calculation at $P_{0}$ requires $\eta=2.92 \times$ $10^{-5}\left(\mathrm{~W} \mathrm{~m}^{-1}\right)^{-1 / 2}$ to obtain $B_{c}=7.35 \mathrm{mT}$. Plotting the time dependence of $P_{\text {nuc }}\left(t, B_{c}\right)$ shows that nucleation occurs when the SAW crosses a threshold compressive strain [Fig. 6(b)], for which the total out-of-plane anisotropy, and therefore the DW energy, are briefly lowered with respect to their base value. This facilitates magnetization switching [13] in a nucleationdominated reversal process, as observed experimentally. With $\eta=2.92 \times 10^{-5}\left(\mathrm{~W} \mathrm{~m}^{-1}\right)^{-1 / 2}$, the coercive fields at all the other powers are calculated, and the resulting curve is shown in Fig. 6(c). The model predicts the coercive field to start decreasing linearly with power for $P_{\mathrm{rf}}>0.4 P_{0}$. The value of $\eta$ determined by this approach corresponds to a maximum transient $K_{u}$ lowering of $31 \%$ (at $P_{0}$ ). It gives a SAW amplitude of $\eta \sqrt{P_{0}}=2.7 \times 10^{-3}$, reasonably about four times larger than the value determined electrically with the VNA.

Although the model is a good qualitative demonstration that the SAW does efficiently assist domain nucleation, it fails to reproduce the experimentally observed slope. The impact of the SAW on DW propagation can probably not be fully neglected in the analysis. It would act through the transient increase of the DW width $\Delta \propto 1 / \sqrt{K_{u}\left(\varepsilon_{\mathrm{SAW}}\right)}$, similar to the observations of Shepley et al. [35] under static strain variations. The probabilistic calculation of SAWassisted nucleation-propagation reversal is beyond the scope of this Rapid Communication, but could, for instance, be treated in the framework derived by Fatuzzo and Labrune for ferroelectric domains $[28,36]$, once the $v(B)$ curve has been determined.

To summarize, we have demonstrated up to $60 \%$ coercivity reduction of a thin out-of-plane magnetized $(\mathrm{Ga}, \mathrm{Mn})(\mathrm{As}, \mathrm{P})$ layer using $549 \mathrm{MHz}$ SAWs. As shown by a rudimentary model, the most likely mechanism involves SAW-assisted domain nucleation, made possible by the transient reduction of the domain wall energy, although it does not entirely account for the great efficiency of the SAW. It is likely the mechanism at play in the data presented by Li et al. in Galfenol [18,19]. What makes this mechanism particularly efficient in $(\mathrm{Ga}, \mathrm{Mn})(\mathrm{As}, \mathrm{P})$ is the low magnetic anisotropy and weak exchange constant of this material, which give a small domain nucleation energy. Lower SAW frequencies would probably make it even more efficient: The SAW amplitude would be higher at equivalent IDT aperture and excitation power, and the layer would have more time per SAW period to nucleate, while profiting from the wave properties of SAWs.

This work was performed in the framework of the SPINSAW project (ANR-13-JS04-0001-01). We acknowledge M. Bernard and S. Majrab for the technical assistance (INSP), J. von Bardeleben (INSP) for the cavity FMR data, and J. Moritz for fruitful discussions. 
[1] A. J. Schellekens, A. van den Brink, J. Franken, H. Swagten, and B. Koopmans, Nat. Commun. 3, 847 (2012).

[2] Y. Shiota, T. Nozaki, F. Bonell, S. Murakami, T. Shinjo, and Y. Suzuki, Nat. Mater. 11, 39 (2011).

[3] S. Cavill, D. E. Parkes, J. Miguel, S. S. Dhesi, K. W. Edmonds, R. P. Campion, and A. W. Rushforth, Appl. Phys. Lett. 102, 032405 (2013).

[4] D. Chiba, M. Sawicki, Y. Nishitani, Y. Nakatani, F. Matsukura, and H. Ohno, Nature (London) 455, 515 (2008).

[5] M. Cormier, V. Jeudy, T. Niazi, D. Lucot, M. Granada, J. Cibert, and A. Lemaître, Phys. Rev. B 90, 174418 (2014).

[6] P. Balestriere, T. Devolder, J.-V. Kim, P. Lecoeur, J. Wunderlich, V. Novak, T. Jungwirth, and C. Chappert, Appl. Phys. Lett. 99, 242505 (2011).

[7] C. Bihler, M. Althammer, A. Brandlmaier, S. Geprägs, M. Weiler, M. Opel, W. Schoch, W. Limmer, R. Gross, M. S. Brandt, and S. T. B. Goennenwein, Phys. Rev. B 78, 045203 (2008).

[8] H. Zhou, A. Talbi, N. Tiercelin, and O. Bou Matar, Appl. Phys. Lett. 104, 114101 (2014).

[9] L. Thevenard, C. Gourdon, J. Y. Prieur, H. J. von Bardeleben, S. Vincent, L. Becerra, L. Largeau, and J.-Y. Duquesne, Phys. Rev. B 90, 094401 (2014).

[10] A. Scherbakov, A. Salasyuk, T. Akazaki, X. Liu, M. Bombeck, C. Brüggemann, D. Yakovlev, V. Sapega, J. Furdyna, and M. Bayer, Phys. Rev. Lett. 105, 117204 (2010).

[11] J. V. Jager, A. Scherbakov, T. L. Linnik, D. R. Yakovlev, M. Wang, P. Wadley, V. Holy, S. Cavill, T. Akazaki, A. W. Rushforth, and M. Bayer, Appl. Phys. Lett. 103, 032409 (2013).

[12] O. Kovalenko, T. Pezeril, and V. V. Temnov, Phys. Rev. Lett. 110, 266602 (2013).

[13] L. Thevenard, J.-Y. Duquesne, E. Peronne, H. J. von Bardeleben, H. Jaffrès, S. Ruttala, J.-M. George, A. Lemaître, and C. Gourdon, Phys. Rev. B 87, 144402 (2013).

[14] L. Thevenard, I. S. Camara, P. Rovillain, A. Lemaitre, C. Gourdon, and J.-Y. Duquesne (unpublished).

[15] S. Davis, A. Baruth, and S. Adenwalla, Appl. Phys. Lett. 97, 232507 (2010).

[16] S. Davis, J. a. Borchers, B. B. Maranville, and S. Adenwalla, J. Appl. Phys. 117, 063904 (2015).

[17] J. Dean, M. T. Bryan, J. D. Cooper, A. Virbule, J. E. Cunningham, and T. J. Hayward, Appl. Phys. Lett. 107, 142405 (2015).
[18] W. Li, B. Buford, A. Jander, and P. Dhagat, IEEE Trans. Magn. 50, 37 (2014).

[19] W. Li, B. Buford, A. Jander, and P. Dhagat, Physica B 448, 151 (2014).

[20] A. Lemaître, A. Miard, L. Travers, O. Mauguin, L. Largeau, C. Gourdon, V. Jeudy, M. Tran, and J. George, Appl. Phys. Lett. 93, 021123 (2008).

[21] T. Dietl and H. Ohno, Rev. Mod. Phys. 86, 187 (2014).

[22] H. Riahi, L. Thevenard, M. Maaref, B. Gallas, A. Lemaître, and C. Gourdon, J. Magn. Magn. Mater. 395, 340 (2015).

[23] L. Thevenard, L. Largeau, O. Mauguin, G. Patriarche, A. Lemaître, N. Vernier, and J. Ferré, Phys. Rev. B 73, 195331 (2006).

[24] H. Maris, in Physical Acoustics, edited by W. P. Mason and R. N. Thurston (Academic, New York, 1971), Vol. VIII, p. 279.

[25] S. Shihab, H. Riahi, L. Thevenard, H. J. von Bardeleben, A. Lemaître, and C. Gourdon, Appl. Phys. Lett. 106, 142408 (2015).

[26] A. P. Malozemofff and J. C. Slonczewski, Magnetic Domain Walls in Bubble Materials (Academic, New York, 1979).

[27] A. Dourlat, V. Jeudy, A. Lemaître, and C. Gourdon, Phys. Rev. B 78, 161303 (2008).

[28] M. Labrune, S. Andrieu, F. Rio, and P. Bernstein, J. Magn. Magn. Mater. 80, 211 (1989).

[29] A. Hubert and R. Schäfer, Magnetic Domains (Springer, Berlin, 2000).

[30] In very soft materials where magnetization reverses before a magnetic field sign change, a demagnetizing contribution must also be included [29] - it will be neglected here given the shape of the hysteresis loop [Fig. 1(c)].

[31] W. F. Brown, Phys. Rev. 130, 1677 (1963).

[32] B. Barbara, J. Magn. Magn. Mater. 129, 79 (1994).

[33] J. Moritz, B. Dieny, J. Nozières, Y. Pennec, J. Camarero, and S. Pizzini, Phys. Rev. B 71, 100402(R) (2005).

[34] D. Royer and E. Dieulesaint, Elastic Waves in Solids I: Free and Guided Propagation, Advanced Texts in Physics (Springer, Berlin, 2000).

[35] P. M. Shepley, A. W. Rushforth, M. Wang, G. Burnell, and T. A. Moore, Sci. Rep. 5, 7921 (2015).

[36] E. Fatuzzo, Phys. Rev. 127, 1999 (1962). 